\title{
La réouverture du Musée de la vie indigène à Léopoldville
}

LE Musée de la vie indigène à Léopoldville vient de réouvrir ses portes, après une fermeture assez prolongée. M. Jean Van den Bossche, conservateur du Musée, a su, avec des moyens des plus réduits, aidé d'un personnel composé seulement de cinq Congolais, tirer un parti heureux de l'espace réduit qui lui est imparti.

Le Musée occupe les anciens locaux de la Poste centrale de Léopoldville. Cependant M. Van den Bossche est arrivé à donner à ces locaux un caractère neuf. On y voit notamment des spécimens de l'art des Bapende, des Bambala, des Bakuba, des Batshoka et des Baluba, etc. De plus, M. Van den Bossche se propose d'organiser, chaque mois, une exposition particuliète et très détaillée se rapportant à une région bien déterminée du Congo et du Ruanda-Urundi.

\section{Film de la F.I.S. sur le Congo Belge}

M. D. BreBuYck a été désigné comme conseiller scientifique de la partie ethnologique du grand film qui sera tourné au Congo Belge sous les auspices de la Fondation Internationale Scientifique. Les différentes scènes, illustrant les relations sociales, tituelles et symboliques entre les hommes et les animaux, seront tournées chez les Nyanga (Kivu-Nord), Lega (Maniéma), Bembe et Shi (Kivu-Sud), et Pygmées (Kivu-Nord). En collaboration avec le Dr. Schäfer, conseiller scientifique, et $\mathrm{M}$. Brandt, réalisateur de la partie ethnologique, M. D. Biebuyck a élaboré le scénario ethnologique.

\section{Les recherches archéologiques et historiques en A.O.F. de 1953 à 1957}

LA situation générale des Instituts de recherches en Afrique noire française n'a pas varié sensiblement depuis 1953: les études historiques et archéologiques restent toujours centrées autour de l'IFAN (Institut Français d'Afrique Noire) dont l'A.O.F. est le champ d'action. Les autres territoires français d'Afrique Noire (Afrique Équatoriale Française, Cameroun, Togo) tout en possédant des Instituts de recherches: l'Institut d'Études Centrafricaines de Brazzaville pour l'A.É.F., et des centres locaux IFAN indépendants de Dakar pour le Cameroun et le Togo, n'ont pas sur place de chercheurs officiels spécialisés dans les questions historiques ou archéologiques.

Les principales missions effectuées depuis 1953 sont les suivantes: Adrar de Mauritanie: Azougui, Chinguetti, Abweir, Ouadane, avec relevé des ruines de la forteresse almoravide d'Azougui près d'Atar et des mosquées anciennes de Chinguetti et d'Ouadane, sans compter des récoltes de préhistoire ( $R$. Mauny, I953); contacts pris dans les Musées et les Instituts de France en vue de la constitution du Musée historique de Gorée (A. Ly, I953); fouilles de l'abri sous roche de Kourinkoro-Kale près de Bamako au Soudan (G. Szumowski, 1953); fouilles dans les environs de Bamako dans les tombeaux protohistoriques (G. Szumowski, 1954); mission dans le Macina et la boucle du Niger avec fouilles dans la région de Goundam dans des tumulus et sur des sites anciens ( $R$. Mauny, 1954-55); fouilles dans les environs de Bamako dans des pseudotumulus et des tombeaux protohistoriques (G. Szumowski, 1955); mission dans la région des mégalithes du Sine-Saloum avec fouilles (R. Mauny, 1956).

La plus grande partie des collections est groupée dans la Section d'Archéologie-Préhistoire et dans la réserve d'Ethnologie de l'IFAN de Dakar.

Des travaux en cours, le principal effort est centré autour de l'utilisation des travaux de recherches déjà effectués (fouilles, prospections, travaux écrits de géographie historique, etc.) en vue de la tédaction d'un 'Tableau géographique de l'Ouest Africain au Moyen Age d'après les sources écrites, la tradition et l'archéologie'. Ce travail couvrirait au point de vue géographique l'ensemble de l'A.O.F. et des territoires étrangers enclavés et, dans le temps, de l'hégire à la découverte portugaise. Les sources écrites sont principalement arabes 